\title{
ASYMPTOTICS OF THE NEGATIVE DISCRETE SPECTRUM OF A CLASS OF SCHRÖDINGER OPERATORS WITH LARGE COUPLING CONSTANT
}

\author{
ARI LAPTEV
}

(Communicated by Barbara L. Keyfitz)

\begin{abstract}
We obtain the asymptotics of the negative discrete spectrum of the Schrödinger operator with a large coupling constant and potentials $V \notin$ $L_{m / 2}\left(R^{m}\right), m \geq 3$. The result is very sensitive to small perturbations of the potential and depends on the negative spectrum of some auxiliary differential problems on $S^{m-1}$.
\end{abstract}

Let us denote by $N(\mu, V)$ the number of negative eigenvalues (including their multiplicities) for the Schrödinger operator

$$
H_{\mu}=-\Delta-\mu V, \quad x \in \mathbf{R}^{m}, m \geq 3, \mu>0 .
$$

If the potential $V \in L_{m / 2}\left(\mathbf{R}^{m}\right)$, then the asymptotic behaviour of $N(\mu, V)$, $\mu \rightarrow \infty$, is well known and is given by the formula

$$
\lim _{\mu \rightarrow \infty} \mu^{-m / 2} N(\mu, V)=v_{m}(2 \pi)^{-m} \int V_{+}^{m / 2} d x,
$$

where $V_{+}$is the positive part of the function $V$ and $v_{m}$ is the volume of the unit ball in $\mathbf{R}^{m}$.

Very little is known, however, about potentials $V \notin L_{m / 2}\left(\mathbf{R}^{m}\right)$, which allows one to obtain an asymptotic formula for the negative spectrum of the operator (1); in fact, there is essentially only one example given in [1], namely,

$$
V_{0} \sim|x|^{-2} \ln ^{-\alpha}|x|, \quad|x| \rightarrow \infty, 2 / \alpha>m .
$$

In this case the following asymptotic formula is valid:

(3) $\lim _{\mu \rightarrow \infty} \mu^{-1 / \alpha} N\left(\mu, V_{0}\right)=\frac{1}{2 \sqrt{\pi}} \frac{\Gamma(1 / \alpha-1 / 2)}{\Gamma(1 / \alpha)} \sum_{j}\left(\Lambda_{j}^{\circ}+\frac{(m-2)^{2}}{4}\right)^{1 / 2-1 / \alpha}$,

where $\Lambda_{j}^{\circ}$ denotes the eigenvalues (including their multiplicities) of the spherical part of the operator $-\Delta$. It is interesting to notice that although $\alpha$ appears

Received by the editors September 9, 1991 and, in revised form, February 11, 1992.

1991 Mathematics Subject Classification. Primary 35P20, 35J10. 
in (2) as a power of $\ln |x|$ (which seemed to not be a very important term), it regulates the main term of the asymptotic formula (3).

Let us now consider the potential

$$
V_{\varphi}(x)=\varphi(x)(\ln 1 / \varphi)^{-\alpha}, \quad 2 / \alpha>m,
$$

where

$$
\varphi(x)= \begin{cases}|x|^{-2} \Psi(\theta), & |x| \geq 1, \\ 0, & |x|<1,\end{cases}
$$

and

$$
\Psi \in L^{m / 2}\left(S^{m-1}\right), \quad \Psi>0 .
$$

From Proposition 3 of [1] (formula (15)) follows the very useful estimate

$$
\sup _{\mu>0} \mu^{-1 / \alpha} N\left(\mu, V_{\varphi}\right) \leq C(\alpha, m)\|\Psi\|_{L^{m / 2}\left(S^{m-1}\right)}^{m / 2} .
$$

The asymptotic formula (3) can be obtained by separating variables for a model problem with a potential equal to $|x|^{-2} \ln ^{-\alpha}|x|$, when $|x| \geq 1$. Then applying the estimate (6) we can "kill" small perturbations converging to zero at infinity faster than the model.

The asymptotic behaviour of the negative spectrum of the Schrödinger operator with a large coupling constant and with potentials of the type (4) and (5) turns out to be important in applications. This problem was posed in [1] as an unsolved problem. The asymptotic formulas given in Theorems 1 and 2 of this paper answer this question.

In this section, we study the asymptotics of the negative spectrum of the operator (1) with potential $V_{\varphi}$ introduced by formulas (4) and (5), where function $\Psi \in L_{\infty}\left(S^{m-1}\right)$ and $\Psi>0$.

We need the following notation. Let $\Delta_{\theta}$ be the Laplace-Beltrami operator on the unit sphere $S^{m-1}$. We shall consider the eigenfunctions $w_{l}(\theta, \nu)$ and the corresponding eigenvalues $\Lambda_{l}(\nu)$ of the differential operator on $S^{m-1}$ :

$$
\left[-\Delta_{\theta}-\nu \Psi(\theta)\right] w_{l}(\theta, \nu)=\Lambda_{l}(\nu) w_{l}(\theta, \nu) .
$$

It is well known that the spectrum of the problem (7) is discrete and $\Lambda_{l}(\nu) \rightarrow$ $+\infty$ when $l \rightarrow \infty$; however, if the value of the parameter $\nu$ is large, then there are a great number of negative eigenvalues.

Let $(t)_{-} \equiv-t$ if $t<0$ and $(t)_{-} \equiv 0$ if $t \geq 0$.

Lemma 1. If $\Psi \in L_{\infty}\left(C^{m-1}\right), \Psi>0, \alpha<2 / m$, then for any constant $a>0$

$$
\sum_{l} \int_{0}^{\infty}\left(\Lambda_{l}\left(t^{-\alpha}\right)+a\right)_{-}^{1 / 2} d t<\infty .
$$

Proof. From variational principles we obtain that

$$
\Lambda_{l}(\nu) \geq \Lambda_{l}^{\circ}-\nu \Psi_{\max }
$$


where $\Psi_{\max }=\max _{\theta \in S^{m-1}} \Psi(\theta)$ and $\Lambda_{l}^{\circ}$ are eigenvalues of the Laplace-Beltrami operator on $S^{m-1}$. This implies

$$
\begin{aligned}
\sum_{l} \int_{0}^{\infty}\left(\Lambda_{l}\left(t^{-\alpha}\right)+a\right)_{-}^{1 / 2} d t & \leq \sum_{l} \int_{0}^{\infty}\left(\Lambda_{l}^{\circ}+a-t^{-\alpha} \Psi_{\max }\right)_{-}^{1 / 2} d t \\
& =\sum_{l}\left(\Lambda_{l}^{\circ}+a\right)^{-1 / \alpha+1 / 2} \Psi_{\max }^{1 / \alpha} \int_{0}^{1} \sqrt{t^{-\alpha}-1} d t .
\end{aligned}
$$

Since $\Lambda_{l}^{\circ} \sim C(m) l^{2 /(m-1)}$ and $\alpha<2 / m, m \geq 3$, we obtain that the value of the right-hand side in (3) is finite. The lemma is proved.

We formulate the first main theorem.

Theorem 1. Let $\Psi(x)=\Psi(x /|x|)>0$ and $\Psi \in L_{\infty}\left(S^{m-1}\right)$. Then the number of negative eigenvalues $N\left(\mu, V_{\varphi}\right)$ for the Schrödinger operator (1) with the potential $V_{\varphi}$ defined by formulas (4), (5) satisfies the asymptotic formula

$$
\begin{aligned}
N\left(\mu, V_{\varphi}\right)= & \mu^{1 / \alpha} \pi^{-1} \sum_{l} \int_{0}^{\infty}\left[\Lambda_{l}\left(t^{-\alpha}\right)+\left(\frac{m-2}{2}\right)^{2}\right]_{-}^{1 / 2} d t \\
& +O\left(\mu^{1 / \alpha-\delta /(1+\alpha \delta)}\right)
\end{aligned}
$$

with $0<\delta<1 / \alpha-m / 2$.

Proof. Let us first simplify the problem. We introduce the quotient of the quadratic forms

$$
Q[u]=\int V_{\varphi}|u|^{2} d x / \int|\nabla u|^{2} d x
$$

The quotient (11) is defined first for $u \in C_{\circ}^{\infty}\left(\mathbf{R}^{m}\right)$ and then the closure is taken in the metric given by the quadratic form in the denominator. Since $m \geq 3$, this closure coincides with the so-called "homogeneous" Sobolev class

$$
\mathscr{H}^{1}=\left\{u \in H_{\mathrm{loc}}^{1}\left(\mathbf{R}^{m}\right): \int\left(|\nabla u|^{2}+|x|^{-2}|u|^{2}\right) d x<\infty\right\} .
$$

The quadratic form $\int V|u|^{2} d x$ in the Hilbert space $\mathscr{H}^{1}\left(\mathbf{R}^{m}\right)$ defines the selfadjoint compact operator. We denote the distribution function of its positive discrete spectrum by $N(s, Q)$ and obtain the relation

$$
N\left(\mu, V_{\varphi}\right)=N(1 / \mu, Q) .
$$

Standard arguments involving variational principles allow us to reduce the problem to the study of the asymptotic behaviour of the distribution function of the spectrum for the quotient

$$
Q_{1}[u]=\frac{\int_{|x| \geq 1}|x|^{-2} \Psi(x /|x|)(\ln |x|)^{-\alpha}|u|^{2} d x}{\int_{|x| \geq 1}|\nabla u|^{2} d x}
$$

where $u \in \mathscr{H}^{1}\left(\mathbf{R}^{m}\right)$ and

$$
\left.u\right|_{|x|=1}=0
$$


If we now introduce the spherical system of coordinates $x=(r, \theta)$ and put $r=e^{t}, u=e^{-(m-2) t / 2} v$, then

$$
Q_{1}[u]=Q_{2}[v]=\frac{\int_{S^{m-1}} \Psi(\theta) d \theta \int_{0}^{\infty} t^{-\alpha}|v|^{2} d t}{\int_{S^{m-1}} \int_{0}^{\infty}\left(\left|v_{t}\right|^{2}+\left|\nabla_{\theta} v\right|^{2}+((m-2) / 2)^{2}|v|^{2}\right) d t d \theta}
$$

and condition (13) becomes the condition

$$
\left.v\right|_{t=0} \text {. }
$$

Now to obtain the theorem we apply the well-known "Dirichlet-Neumann bracketing" (see, e.g., [3]), using it with respect to the variable $t \in(0, \infty)$.

Let $\varepsilon>0$. We introduce the quotient

$$
\frac{\int_{S^{m-1}} \int_{k \varepsilon}^{(k+1) \varepsilon} \Psi(\theta) t^{-\alpha}|v|^{2} d t d \theta}{\int_{S^{M-1}} \int_{k \varepsilon}^{(k+1) \varepsilon}\left(\left|v_{t}\right|^{2}+\left|\nabla_{\theta} v\right|^{2}+((m-2) / 2)^{2}|v|^{2}\right) d t d \theta} .
$$

Denote the quotient (16) by $Q_{k \varepsilon}^{D}[v], k=0,1, \ldots$, and $Q_{k \varepsilon}^{N}[v], k=1,2, \ldots$, if it is defined on the functions $v \in H^{1}\left(S^{m-1} \times(k \varepsilon,(k+1) \varepsilon)\right)$ with Dirichlet and Neumann boundary conditions respectively. If $k=0$, then by $Q_{0 \varepsilon}^{N}[v]$ we mean the quotient (16) defined on the functions with Neumann boundary condition on the "top" part of the boundary $S^{m-1} \times\{\varepsilon\}$ and Dirichlet boundary condition on the "bottom" $S^{m-1} \times\{0\}$.

By analogy we also introduce the quotient

$$
\frac{\int_{S^{m-1}} \int_{k \varepsilon}^{(k+1) \varepsilon} \Psi(\theta)|v|^{2} d t d \theta}{\int_{S^{m-1}} \int_{k \varepsilon}^{(k+1) \varepsilon}\left(\left|v_{t}\right|^{2}+\left|\nabla_{\theta} v\right|^{2}+((m-2) / 2)^{2}|v|^{2}\right) d t d \theta}
$$

and correspondingly its values $R_{k \varepsilon}^{D}[v]$ and $R_{k \varepsilon}^{N}[v]$ for $k=1,2, \ldots$

Now the "Dirichlet-Neumann bracketing" gives us the estimates

$$
\begin{gathered}
N\left(s, Q_{2}\right) \leq \sum_{k=0}^{\infty} N\left(s, Q_{k \varepsilon}^{N}\right) \leq N\left(s, Q_{0 \varepsilon}^{N}\right)+\sum_{k=1}^{\infty} N\left(s,(k \varepsilon)^{-\alpha} R_{k \varepsilon}^{N}\right), \\
N\left(s, Q_{2}\right) \geq \sum_{k=0}^{\infty} N\left(s, Q_{k \varepsilon}^{D}\right) \geq \sum_{k=0}^{\infty} N\left(s,((k+1) \varepsilon)^{-\alpha} R_{k \varepsilon}^{D}\right) .
\end{gathered}
$$

Let $n_{k \varepsilon}^{D}(\nu)(k=0,1, \ldots)$ and $n_{k \varepsilon}^{N}(\nu)(k=1,2, \ldots)$ be the distribution functions of the negative spectrum of the operators $A_{k \varepsilon}^{D}(\nu)$ and $A_{k \varepsilon}^{N}(\nu)$ defined by the quadratic form

$$
\int_{S^{m-1}} \int_{k \varepsilon}^{(k+1) \varepsilon}\left(\left|v_{t}\right|^{2}+\left|\nabla_{\theta} v\right|^{2}+\left(\frac{m-2}{2}\right)^{2}|v|^{2}-\nu \Psi|v|^{2}\right) d t d \theta
$$

with Dirichlet and Neumann boundary conditions respectively. We obtain the relations

$$
\begin{gathered}
N\left(s,(k \varepsilon)^{-\alpha} R_{k \varepsilon}^{N}\right)=n_{k \varepsilon}^{N}\left(s^{-1}(k \varepsilon)^{-\alpha}\right), \\
N\left(s,((k+1) \varepsilon)^{-\alpha} R_{k \varepsilon}^{D}\right)=n_{k \varepsilon}^{D}\left(s^{-1}((k+1) \varepsilon)^{-\alpha}\right) .
\end{gathered}
$$

The eigenfunctions of the operators $A_{k \varepsilon}^{N}(\nu)$ and $A_{k \varepsilon}^{D}(\nu)$ are equal to

$$
\cos \frac{\pi n(t-k \varepsilon)}{\varepsilon} w_{l}(\theta, \nu), \quad n=0,1,2, \ldots, l=1,2, \ldots,
$$




$$
\sin \frac{\pi n(t-k \varepsilon)}{\varepsilon} w_{l}(\theta, \nu), \quad n=1,2, \ldots, l=1,2, \ldots,
$$

where $w_{l}(\theta, \nu)$ were introduced by formula (7). The corresponding eigenvalues are equal to

$$
\left(\frac{\pi}{\varepsilon}\right)^{2} n^{2}+\Lambda_{l}(\nu)+\left(\frac{m-2}{2}\right)^{2},
$$

where $\Lambda_{l}(\nu)$ are eigenvalues of the differential operator $T_{\nu}=-\Delta_{\theta}-\nu \Psi$ on $S^{m-1}$.

(a) Let us first study the inequality (17). To estimate the term $N\left(s, Q_{0 \varepsilon}^{N}\right)$ in (17) we need the following statement (see [4]).

Lemma 2. Let $\Omega \subset \mathbf{R}^{m}$ be an open domain, $b \in L_{m / 2}(\Omega), b \geq 0, m \geq 3$. The quadratic form $\int_{\Omega} b|v|^{2} d x$ in Hilbert space $H_{\circ}^{1}(\Omega)$ defines the selfadjoint positive compact operator, and for the distribution function $N(s)$ of its discrete spectrum the following inequality holds:

$$
N(s) \leq C s^{-m / 2} \int_{\Omega}|b|^{m / 2} d x,
$$

where the constant $C$ is independent of $\Omega$ and $b$.

Lemma 2 implies the inequality

$$
\begin{aligned}
N\left(s, Q_{0 \varepsilon}^{N}\right) & \leq N\left(s, Q_{0(2 \varepsilon)}^{D}\right) \\
& \leq C s^{-m / 2} \int_{0}^{2 \varepsilon} \int_{S^{m-1}}\left[\Psi(\theta) t^{-\alpha}\right]^{m / 2} d \theta d t \leq C^{\prime} s^{-m / 2} \varepsilon^{1-m \alpha / 2},
\end{aligned}
$$

where the constant $C^{\prime}$ is independent of $s$ and $\varepsilon$.

Since the eigenvalues of the operator $A_{k \varepsilon}^{N}(\nu)$ are equal to (22), we obtain

$$
\begin{aligned}
\sum_{k=1}^{\infty} N\left(s,(k \varepsilon)^{-\alpha} R_{k \varepsilon}^{N}\right)= & \sum_{k=1}^{\infty} n_{k \varepsilon}^{N}\left(s^{-1}(k \varepsilon)^{-\alpha}\right) \\
= & \sum_{n=0}^{\infty} \sum_{k, l=1}^{\infty} 1:\left\{\left(\frac{\pi}{\varepsilon}\right)^{2} n^{2}+\Lambda_{l}\left(s^{-1}(k \varepsilon)^{-\alpha}\right)+\left(\frac{m-2}{2}\right)^{2}<0\right\} \\
\leq & \frac{\varepsilon}{\pi} \sum_{k, l=1}^{\infty}\left(\Lambda_{l}\left(s^{-1}(k \varepsilon)^{-\alpha}\right)+\left(\frac{m-2}{2}\right)\right)^{1 / 2} \\
& +\sum_{k, l=1}^{\infty} 1:\left\{\Lambda_{l}\left(s^{-1}(k \varepsilon)^{-\alpha}\right)+\left(\frac{m-2}{2}\right)^{2}<0\right\} .
\end{aligned}
$$

Now let us notice that the terms of the sum on the right-hand side of the last inequality are not equal to zero only if

$$
\Lambda_{l}\left(s^{-1}(k \varepsilon)^{-\alpha}\right)+\left(\frac{m-2}{2}\right)^{2}<0 .
$$

This gives the estimate

$$
\Lambda_{l}^{\circ}-s^{-1}(k \varepsilon)^{-\alpha} \Psi_{\max }+\left(\frac{m-2}{2}\right)^{2}<0,
$$


which leads to

$$
k<s^{-1 / \alpha} \varepsilon^{-1} \Psi_{\max }^{1 / \alpha}\left[\Lambda_{l}^{\circ}+\left(\frac{m-2}{2}\right)^{2}\right]^{-1 / \alpha}
$$

and for $k=1$,

$$
s<\varepsilon^{-\alpha} \Psi_{\max }\left[\Lambda_{l}^{\circ}+\left(\frac{m-2}{2}\right)^{2}\right]^{-1} .
$$

From (25) we obtain the following estimate for $\left(24^{\prime}\right)$

$$
\begin{aligned}
& \sum_{k, l=1}^{\infty} 1:\left\{\Lambda_{l}\left(s^{-1}(k \varepsilon)^{-1 / \alpha}\right)+\left(\frac{m-2}{2}\right)^{2}<0\right\} \\
& \leq C s^{-1 / \alpha} \varepsilon^{-1} \sum_{l=1}^{\infty}\left(\Lambda_{l}^{\circ}+\left(\frac{m-2}{2}\right)^{2}\right)^{-1 / \alpha} \leq C^{\prime} \varepsilon^{-1} s^{-1 / \alpha} .
\end{aligned}
$$

It is clear that for the term (24) we obtain

$$
\begin{aligned}
& \frac{\varepsilon}{\pi} \sum_{k, l=1}^{\infty}\left(\Lambda_{l}\left(s^{-1}(k \varepsilon)^{-\alpha}\right)+\left(\frac{m-2}{2}\right)^{2}\right)_{-}^{1 / 2} \\
& \quad \leq \frac{1}{\pi} \sum_{l=1}^{\infty} \int_{0}^{\infty}\left(\Lambda_{l}\left(s^{-1} t^{-\alpha}\right)+\left(\frac{m-2}{2}\right)^{2}\right)_{-}^{1 / 2} d t+\frac{\varepsilon}{\pi} \sum_{k, l=1}^{\infty} M_{k l}(s, \varepsilon),
\end{aligned}
$$

where

$$
M_{k l}(s, \varepsilon)=\sup _{t_{1}, t_{2} \in[k \varepsilon,(k+1) \varepsilon)}\left|\Lambda_{l}\left(s^{-1} t_{1}^{-\alpha}\right)-\Lambda_{l}\left(s^{-1} t_{2}^{-\alpha}\right)\right|^{1 / 2}
$$

It is obvious that

$$
\begin{aligned}
M_{k l}(s, \varepsilon) & \leq \| T_{s^{-1} t_{1}^{-\alpha}}-T_{s^{-1} t_{2}^{-\alpha} \|^{1 / 2}} \\
& \leq C\left(s^{-1} \varepsilon^{-\alpha} k^{-\alpha}\right)^{1 / 2} k^{-1}=C \varepsilon^{-\alpha / 2} k^{-\alpha / 2-1} s^{-1 / \alpha+\delta} s^{1 / \alpha-\delta-1 / 2}
\end{aligned}
$$

Let $0<\delta<1 / \alpha-m / 2$. The estimate (26) implies

$$
\begin{aligned}
\frac{\varepsilon}{\pi} \sum_{k, l=1}^{\infty} M_{k, l}(s, \varepsilon) \leq C \frac{\varepsilon}{\pi} \sum_{k, l=1}^{\infty} \varepsilon^{-1+\alpha \delta} s^{(\delta-1 / \alpha)} k^{-\alpha / 2-1} & \\
& \times\left(\Lambda_{l}^{\circ}+\left(\frac{m-2}{2}\right)^{2}\right)^{1 / 2-1 / \alpha+\delta} \\
& \leq C^{\prime} \varepsilon^{\alpha \delta} s^{\delta-1 / \alpha}
\end{aligned}
$$

where $C^{\prime}$ is independent of $s$ and $\varepsilon$. Applying (23), (27), (28), and (30) to (17) we obtain

$$
\begin{aligned}
N\left(s, Q_{2}\right) \leq & \frac{1}{\pi} \sum_{l=1}^{\infty} \int_{0}^{\infty}\left(\Lambda_{l}\left(s^{-1} t^{-\alpha}\right)+\left(\frac{m-2}{2}\right)^{2}\right)_{-}^{1 / 2} d t \\
& +C\left(s^{-m / 2} \varepsilon^{1-m \alpha / 2}+s^{-1 / \alpha} \varepsilon^{-1}+\varepsilon^{\alpha \delta} s^{\delta-1 / \alpha}\right)
\end{aligned}
$$


with some constant independent of $s$ and $\varepsilon$. Let us take $\varepsilon=\tilde{\varepsilon} s^{-\gamma}, 0<\gamma<1$. Then

$$
\begin{aligned}
N\left(s, Q_{2}\right) \leq & s^{-1 / \alpha} \frac{1}{\pi} \sum_{l} \int_{0}^{\infty}\left(\Lambda_{l}\left(s^{-1} t^{-\alpha}\right)+\left(\frac{m-2}{2}\right)^{2}\right)_{-}^{1 / 2} d t \\
& +O\left(s^{-m / 2-\gamma(1-m \alpha / 2)}+s^{-1 / \alpha+\gamma}+s^{\delta-1 / \alpha-\alpha \delta \gamma}\right),
\end{aligned}
$$

when $s \rightarrow 0$. The equation $-1 / \alpha+\gamma=\delta-1 / \alpha-\alpha \delta \gamma$ implies $\gamma=\delta /(1+\alpha \delta)$. Now since

$$
-\frac{m}{2}-\frac{\delta}{1+\alpha \delta}\left(1-\frac{m}{2} \alpha\right)>-\frac{1}{\alpha}+\frac{\delta}{1+\alpha \delta} \text { for } 0<\delta<\frac{1}{\alpha}-\frac{m}{2},
$$

we finally obtain

$$
N\left(s, Q_{2}\right) \leq s^{-1 / \alpha} \frac{1}{\pi} \sum_{l} \int_{0}^{\infty}\left(\Lambda_{l}\left(t^{-\alpha}\right)+\left(\frac{m-2}{2}\right)^{2}\right)_{-}^{1 / 2} d t+O\left(s^{-1 / \alpha+\delta /(1+\alpha \delta)}\right)
$$

(b) The lower bound holds by an analogous construction. It follows from (18) and (21) that

$$
\begin{aligned}
N\left(s, Q_{2}\right) \geq & \sum_{k=0}^{\infty} N\left(s,((k+1) \varepsilon)^{-\alpha} R_{k \varepsilon}^{D}\right)=\sum_{k=0}^{\infty} n_{k \varepsilon}^{D}\left(s^{-1}((k+1) \varepsilon)^{-\alpha}\right) \\
\geq & \sum_{k=0}^{\infty} \sum_{l, n=0}^{\infty} 1:\left\{\left(\frac{\pi}{\varepsilon}\right)^{2}+\Lambda_{l}\left(s^{-1}((k+1) \varepsilon)^{-\alpha}\right)+\left(\frac{m-2}{2}\right)^{2}<0\right\} \\
\geq & \frac{\varepsilon}{\pi} \sum_{k=0}^{\infty} \sum_{l=1}^{\infty}\left(\Lambda_{l}\left(s^{-1}((k+1) \varepsilon)^{-\alpha}\right)+\left(\frac{m-2}{2}\right)^{2}\right)_{-}^{1 / 2} \\
& -\sum_{k=0}^{\infty} 1:\left\{\Lambda_{l}\left(s^{-1}((k+1) \varepsilon)^{-\alpha}\right)+\left(\frac{m-2}{2}\right)^{2}<0\right\} .
\end{aligned}
$$

By analogy with (28)

$$
\begin{aligned}
& \frac{\varepsilon}{\pi} \sum_{k=0}^{\infty} \sum_{l=1}^{\infty}\left(\Lambda_{l}\left(s^{-1}((k+1) \varepsilon)^{-\alpha}\right)+\left(\frac{m-2}{2}\right)^{2}\right)_{-}^{1 / 2} \\
& \quad \geq \frac{1}{\pi} \sum_{l=1}^{\infty} \int_{0}^{\infty}\left(\Lambda_{l}\left(s^{-1} t^{-\alpha}\right)+\left(\frac{m-2}{2}\right)^{2}\right)_{-}^{1 / 2} d t-\sum_{k=0}^{\infty} \sum_{l=1}^{\infty} M_{(k+1) l}(s, \varepsilon) .
\end{aligned}
$$

Inequalities (27) and (30) with $\varepsilon=\tilde{\varepsilon} s^{-\gamma}, \gamma=\delta /(1+\alpha \delta)$, yield

$$
N\left(s, Q_{2}\right) \geq s^{-1 / \alpha} \frac{1}{\pi} \sum_{l} \int_{0}^{\infty}\left(\Lambda_{l}\left(t^{-\alpha}\right)+\left(\frac{m-2}{2}\right)^{2}\right)_{-}^{1 / 2} d t+O\left(s^{-1 / \alpha+\delta(1+\alpha \delta)}\right) .
$$

The theorem is proved.

Theorem 1 together with the inequality (6) allow us to consider a "closure" of the class of potentials (4) and (5) with $\Psi \in L_{\infty}\left(S^{m-1}\right)$ in the class $L_{m / 2}\left(S^{m-1}\right)$ (see [2]). 
Theorem 2. Under the conditions of Theorem 1 the following asymptotic formula holds for $\Psi \in L_{m / 2}\left(S^{m-1}\right)$ :

$$
\lim _{\mu \rightarrow \infty} \mu^{-1 / \alpha} N\left(\mu, V_{\varphi}\right)=\pi^{-1} \sum_{l} \int_{0}^{\infty}\left[\Lambda_{l}\left(t^{-\alpha}\right)+\left(\frac{m-2}{2}\right)^{2}\right]_{-}^{1 / 2} d t .
$$

Let us consider a differential operator

$$
H f=-\operatorname{div}(B \operatorname{grad} f)-\mu V_{\circ} f, \quad x \in \mathbf{R}^{m}, m \leq 3, \mu>0,
$$

where $B$ is a constant positive definite matrix and $V_{\circ}$ is defined in (2).

Let $U$ be a unitary operator in $L_{2}\left(\mathbf{R}^{m}\right)$ defined by the formula

$$
f(x)=(U g)(x)=B^{-1 / 4} g\left(B^{-1 / 2} x\right) .
$$

Then

$$
\left(U^{*} H U g\right)(x)=-\Delta g(x)-\mu V_{\circ}\left(B^{1 / 2} x\right) g(x) .
$$

The eigenvalues of the operators $H$ and $U^{*} H U$ coincide.

By analogy with the formula (7) we introduce the following differential operator on $S^{m-1}$ :

$$
-\Delta_{\theta}-\nu(B x, x) /|x|^{2} \text {. }
$$

Let $\Lambda_{l}(\nu, B)$ be the eigenvalues for this operator. Theorem 1 implies

Theorem 3. The asymptotics of the number of the negative eigenvalues $N(\mu, H)$ of the operator (28) is given by

$N(\mu, H)=\mu^{1 / \alpha} \pi^{-1} \sum_{l} \int_{0}^{\infty}\left(\Lambda_{l}\left(t^{-\alpha}, B\right)+\left(\frac{m-2}{2}\right)^{2}\right)_{-}^{1 / 2} d t+O\left(\mu^{1 / \alpha-\delta /(1+\alpha \delta)}\right)$

with $0<\delta<1 / \alpha-m / 2$.

\section{ACKNOWLEDGMENT}

I am grateful to M. Sh. Birman who attracted my attention to this problem. I am also grateful to M. Sh. Birman and Yu. Safarov for useful discussion and to B. L. Keyfitz for correcting and improving the text.

\section{REFERENCES}

1. M. Sh. Birman and M. Z. Solomyak, Negative discrete spectrum of the Schrödinger operator with large coupling constant: A qualitative discussion, Operator Theory: Adv. Appl., vol. 46, Birkhäuser, Basel and Boston, 1990.

2. __ Spectral theory of self-adjoint operators in Hilbert space, Reidel, Dordrecht, 1986.

3. M. Reed and B. Simon, Methods of modern mathematical physics, vol. 4, Academic Press, New York, 1978.

4. G. V. Rozenblum, On the eigenvalues of the first boundary problem in unbounded domains, Math. USSR-Sb. 89 (1972), 234-247.

Department of Mathematics, Linkoping University, S-581 83 Linkoping, SWeden E-mail address: arlap@math.liu.se 